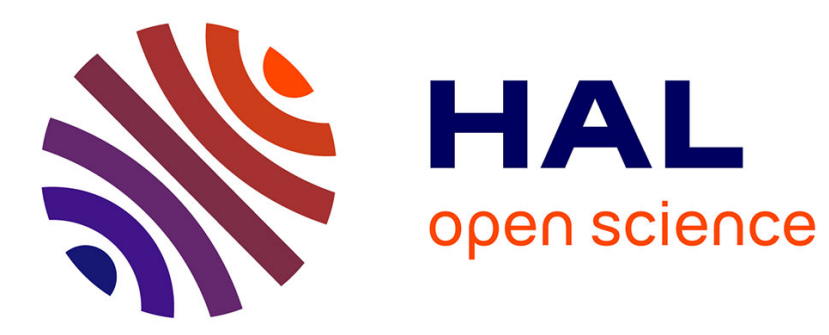

\title{
Étude des rayonnements émis par 126I
}

Nicolas C.M. Marty, H. Langevin, P. Hubert

\section{To cite this version:}

Nicolas C.M. Marty, H. Langevin, P. Hubert. Étude des rayonnements émis par 126I. J. Phys. Radium, 1953, 14 (12), pp.663-671. 10.1051/jphysrad:019530014012066300 . jpa-00234824

\section{HAL Id: jpa-00234824 https://hal.science/jpa-00234824}

Submitted on 1 Jan 1953

HAL is a multi-disciplinary open access archive for the deposit and dissemination of scientific research documents, whether they are published or not. The documents may come from teaching and research institutions in France or abroad, or from public or private research centers.
L'archive ouverte pluridisciplinaire HAL, est destinée au dépôt et à la diffusion de documents scientifiques de niveau recherche, publiés ou non, émanant des établissements d'enseignement et de recherche français ou étrangers, des laboratoires publics ou privés. 


\title{
ÉTUDE DES RAYONNEMENTS ÉMIS PAR ${ }^{126 I}$
}

\author{
Par Mmes N. MARTY, H. LANGEVIN, \\ Laboratoire de Chimie nucléaire du Collège de France, \\ et M. P. HUBERT (*), \\ Laboratoire de Synthèse atomique, Ivry,
}

\begin{abstract}
Sommaire. - Les différents rayonnements émis lors de la désintégration de ${ }^{126}$ I ont été étudiés au moyen d'un spectromètre à lentille magnétique et d'une installation de compteurs à scintillation. On a trouvé deux spectres $\beta^{-}$d'énergie $(\mathrm{r}, 255 \pm 0,01) \mathrm{MeV}$ et $(0,87 \pm 0,02) \mathrm{MeV}$, un spectre $\beta^{+}$ d'énergie $(\mathrm{r}, 2 \mathrm{I} \pm 0,05) \mathrm{MeV}$. On a mis en évidence deux rayonnements $\gamma$ de 386 et $670 \mathrm{keV}$ d'intensités sensiblement égales et un rayonnement $\mathrm{X}$ provenant du tellure indiquant l'existence de deux captures électroniques importantes. Des mesures en coïncidence ont permis d'établir un schéma de désintégration cohérent compte tenu des spins et des parités des différents niveaux. On trouve notamment $J=\mathrm{I}^{+}$pour l'état fondamental de ${ }^{126} \mathrm{I}$.
\end{abstract}

Historique. - ${ }^{126}$ I de période $\mathbf{\imath} 3$ jours a déjà fait l'objet de deux études assez détaillées; les premiers, Mitchell et ses collaborateurs [1], irradiant de l'antimoine par des particules $\alpha$ de $23 \mathrm{MeV}$ obtinrent un mélange de ${ }^{123} \mathrm{I}$ de période $\mathrm{r} 3 \mathrm{~h}$, de ${ }^{124} \mathrm{I}$ de période 4 jours et de ${ }^{126} \mathrm{I}$ de période 13 jours; une étude de l'ensemble avec un spectromètre à lentille magnétique et un spectromètre à focalisation semi-circulaire qui séparait les électrons positifs émis par ${ }^{124} \mathrm{I}$ des électrons négatifs émis par ${ }^{126} I$ conduisit à attribuer à ${ }^{126} \mathrm{I}$ deux spectres $\beta$ - d'énergies maxima 1,268 MeV (27 pour 100) et o,85 MeV (73 pour roo) ainsi qu'un rayonnement $\gamma$ partiellement converti de $395 \mathrm{keV}$.

Perlman et Friedlander [2] préparant ${ }^{126} \mathrm{I}$ par réaction $(n, 2 n)$ sur ${ }^{127} \mathrm{I}$ confirment les résultats précédents relatifs à l'émission $\beta$ - et au photon $\gamma$ pour lequel ils donnent une énergie de $382 \mathrm{keV}$; ils mettent en évidence au compteur proportionnel une forte capture $\mathrm{K} \mathrm{de}{ }^{126} \mathrm{I}$ qui en tenant compte de la capture $L$ probable donne le rapport

$$
\frac{\text { Capture électronique }}{\beta-}=\mathbf{1}, 44 \text {. }
$$

Avec un spectromètre à scintillation, ils trouvent un photon d'intensité faible d'environ $640 \mathrm{keV}$ et par comparaison des coïncidences $\gamma \gamma$ à $180^{\circ}$ et $135^{\circ}$ ils indiquent une proportion de 2 pour 1 oo d'électrons positifs; en outre, ils signalent l'existence de coïncidences $X \gamma$ et $\gamma \gamma$.

Il nous a semblé intéressant de reprendre l'étude de ${ }^{126}$ I pour préciser ses différents modes de désintégration, pour essayer de mettre en évidence la forme des spectres $\beta$ - classés [3] d'après les modèles en couche dans le groupe $\Delta \mathrm{I}=\mathrm{I}$, non $\Delta l=2$ dit " interdit en $l$ ) dont les spectres $\beta$ ont une forme voisine de la forme permise et une valeur de $f t$ corres-

(*) Actuellement au C. E. A., Saclay. pondant à une transition une fois interdite. Enfin nous avons essayé de voir si nous pouvions mesurer le coefficient de conversion du photon de $390 \mathrm{keV}$ avec une précision suffisante pour définir sans ambiguïté sa nature et son ordre de multipolarité.

Une partie des résultats obtenus au cours de ce travail a été publiée dans une Note aux Comptes rendus de l'Académie des Sciences [4].

Préparation. - ${ }^{126}$ I était obtenu par réaction $(d, d n)$ sur ${ }^{127}$ I en irradiant de l'iodure de sodium avec des deutons de $28 \mathrm{MeV}$ au synehrocyclotron Philips d'Amsterdam; cette préparation, mise au point par le Professeur A. H. W. Aten Jr. $\left(^{1}\right)$ conduit à des produits d'activité spécifique de 0,2 à $0,3 \mu \mathrm{C} / \mathrm{mg}$, la meilleure source ayant une activité de $5 \mu \mathrm{C}$ pour $15 \mathrm{mg}$ d'I. Avant son envoi, I était séparé de $\mathrm{Na}$ très actif, par oxydation et extraction par $\mathrm{CCl}_{4}$, ensuite il est réextrait par une solution de $\mathrm{So}_{3} \mathrm{Na}_{2}$. $\mathrm{Au}$ laboratoire, M. Süe a séparé I de $\mathrm{SO}_{3} \mathrm{Na}_{2}$ en traitant la solution par $\mathrm{NO}_{3} \mathrm{Na}$ et $\mathrm{NO}_{3} \mathrm{H}$ normal. I libéré était extrait par $\mathrm{CCl}_{4}$, puis séparé de $\mathrm{CCl}_{4}$ par une solution saturée de $\mathrm{SH}_{2}$ et titré; on chassait $\mathrm{SH}_{2}$ en excès.

La période d'un échantillon suivie pendant plus d'un mois nous a donné la valeur $13,0 \pm 0,25$ jours en bon accord avec la valeur de i 3 jours donnée jusqu'à présent.

Nous avons étudié les rayonnements émis par ${ }^{126} I$ avec un spectromètre à lentille magnétique et un spectromètre à scintillation, nous décrivons successivement les résultats obtenus par ces deux méthodes.

1. Étude au spectromètre à lentille magnétique. - Caractéristiques DE L'APPAREIL. - Nous avons utilisé le spectromètre à lentille magnétique

(1) Nous tenons à le remercier ici tout particulièrement, ainsi que l'équipe du cyclotron Philips pour les différentes sources qu'il a bien voulu nous faire parvenir. 
épaisse construit par l'un de nous [5], [6] au Laboratoire de Synthèse atomique d'Ivry. Étant donné leur faible activité spécifique, les sources déposées sur des feuilles de formvar aluminé d'environ $50 \mu \mathrm{g} / \mathrm{cm}^{2}$ avaient des diamètres de $\mathrm{I} \mathrm{cm}$ entraînant pour le spectromètre un pouvoir de résolution de 3,3 pour 100 et un pouvoir collecteur de i,8 pour ıoo. Un système de diaphragmes hélicoïdaux permettait de séparer les électrons positifs des électrons négatifs; on a vérifié, au cours d'études précédentes, que ces diaphragmes ne déformaient pas les spectres de façon appréciable et qu'avec des sources très intenses émettrices $\beta-$, le nombre d'électrons diffusés et comptés comme électrons positifs était inférieur à $\mathrm{IO}^{-3} \mathrm{du}$ nombre des électrons négatifs. Le détecteur était un compteur cloche à fenêtre de mica de $\mathrm{I}, 7 \mathrm{mg} / \mathrm{cm}^{2}$.

RAPPORT $\frac{\beta^{+}}{\beta-}$ - Avec une première source épaisse d'environ $8 \mathrm{mg} / \mathrm{cm}^{2}$, nous avons pu mettre en évidence le spectre d'électrons positifs représenté figure I; la droite de Fermi correspondante donne une énergie maxima $E_{1}^{+}=\mathrm{I,21} \pm 0,05 \mathrm{MeV}$. L'écart par rapport à la droite apparaît pour une énergie d'environ $0,5 \mathrm{MeV}$; il peut être dû̀ à un deuxième spectre $\beta^{+}$, mais peut s'expliquer seulement par la diffusion des électrons dans la source épaisse. Une
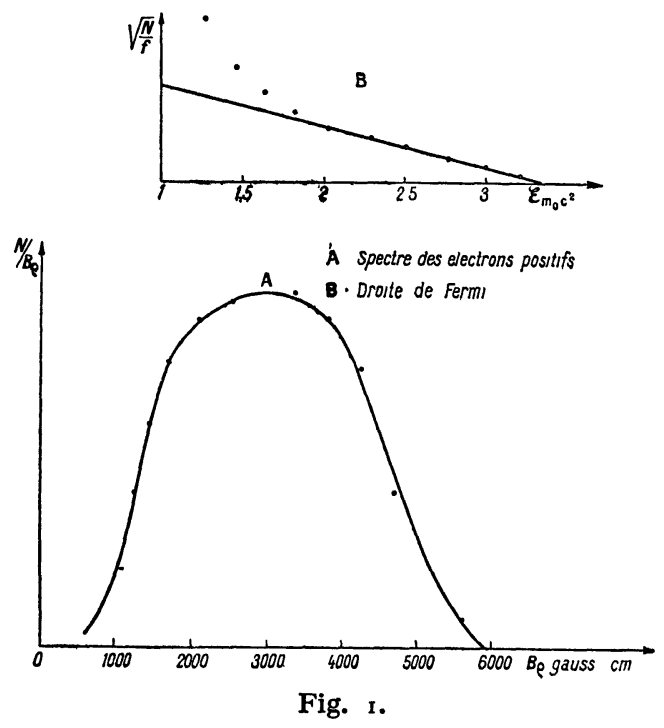

mesure de la forme du spectre $\beta$ - avec la même source conduit au résultat

$$
\frac{\text { Nombre de } \beta+}{\text { Nombre de } \beta-}=0,027 \pm 0,002,
$$

ce rapport représentant le rapport des surfaces des spectres expérimentaux $\beta^{+}$et $\beta^{-}$.

Forme des spectres $\beta$-. - Avec une source d'environ $2 \mathrm{mg} / \mathrm{cm}^{2}$, nous avons étudié de façon plus précise la forme du spectre $\beta^{-}$. La courbe figure 2 est tracée en prenant pour chaque point la moyenne arithmétique des valeurs obtenues au cours de plusieurs séries de mesures. En décomposant le spectre total par la méthode des droites de Fermi,

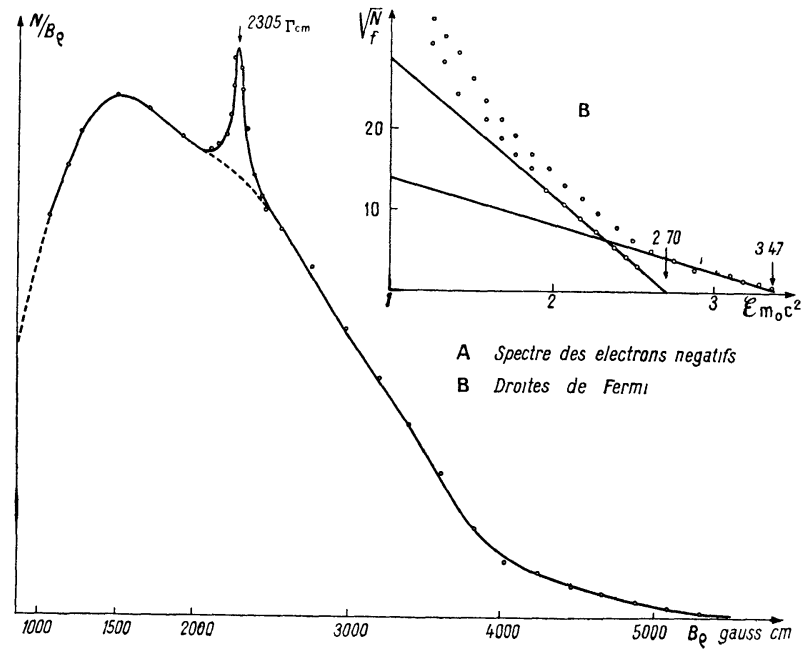

Fig. 2.

on obtient deux spectres partiels d'énergies maxima $E_{1}=1,255 \pm 0$,o $\mathrm{MeV}$ et $E_{2}=0,87 \mathrm{MeV}$, qui semblent à la précision des mesures près avoir une forme permise. Leurs intensités relatives déduites après recomposition des spectres théoriques sont 27,5 et 72,5 pour ioo. L'écart par rapport à la droite de Fermi commence vers $48 \mathrm{o} \mathrm{keV}$ et peut s'expliquer par l'épaisseur de la source.

Élegtrons de conversion. - La raie d'électrons de conversion visible sur la figure 2 est représentée agrandie figure 3 , son énergie $350 \pm 2,5 \mathrm{keV}$ $\left(B_{\rho}=2305 \Gamma \mathrm{cm}\right)$ correspond à un photon de $384,6 \pm 2,5 \mathrm{keV}$ converti dans la couche $K$ de ${ }_{54}^{126} \mathrm{Xe}$ auquel aboutit ${ }^{126} I$ par désintégration $\beta-$. La raie de conversion $L$ de ce rayon $\gamma$ d'énergie $379, \mathbf{1 ~ k e V}$ devrait apparaître pour une valeur $B_{\rho}=2427 \Gamma \mathrm{cm}$; plusieurs séries de mesures ne nous ont pas permis de mettre en évidence dans cette région une raie ayant une intensité dépassant les fluctuations; ceci conduit à un rapport $\frac{\alpha_{K}}{\alpha_{L}}<8, \alpha_{K}$ et $\alpha_{L}$ étant les coefficients de conversion du photon dans les couches $K$ et $L$.

La raie de conversion a une largeur à mi-hauteur de 4,2 pour roo supérieure au pouvoir de résolution. du spectromètre utilisé pour cette mesure; cet élargissement est dû à l'épaisseur de la source qui a aussi pour effet de déplacer le maximum de la raie vers les basses énergies [7]. Si l'on tient compte de cette correction, l'énergie du photon est $386 \pm 2,5 \mathrm{keV}$. En supposant ce rayon $\gamma$ émis en cascade avec le 
spectre $\beta$ - le plus mou $\left(E_{1}-E_{2}=385 \mathrm{keV}\right)$, ce que nous avons vérifié par la suite par coïncidences $\beta \gamma$, on peut en déduire le coefficient de conversion $\alpha_{K}$ du photon d'après le rapport des surfaces de la raie de conversion et du spectre d'énergie maxima $870 \mathrm{keV}$, la surface de ce spectre étant prise comme égale à 72,5 pour ı oo de la surface totale du spectre expérimental; on obtient la valeur

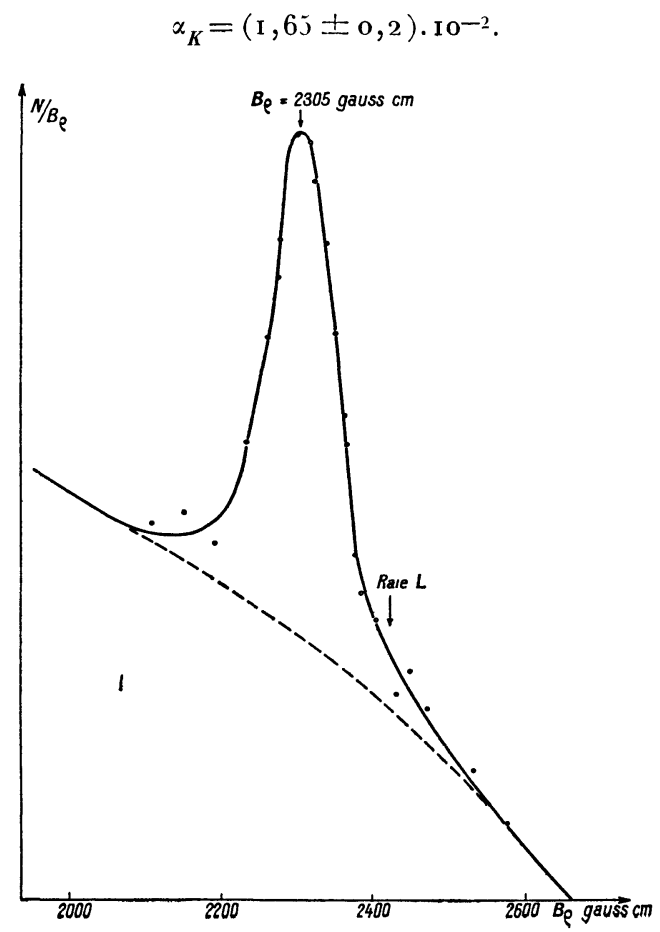

Fig. 3.

ÉTUDE DES RAYONNEMENTS $\gamma$ PAR EFFET PHOTOÉLECTRIQUE. - Par effet photoélectrique, en utilisant un diffuseur de $\mathrm{Au}$ de $30 \mathrm{mg} / \mathrm{cm}^{2}$, nous avons $\mathrm{pu}$ mettre en évidence deux $\gamma$ d'énergie $393 \pm 6 \mathrm{keV}$ et $662 \pm 12 \mathrm{keV}$, d'intensités sensiblement égales; la précision de cette mesure était faible, car avec la source utilisée, la raie de $662 \mathrm{keV}$ par exemple correspondait à un maximum de 20 coups net $/ \mathrm{mn}$ pour un mouvement propre de 12 coups $/ \mathrm{mn}$.

2. Étude au spectromètre à scintillation. DESGRIPTION DU SPECTROMÈTRE. - Nous avons utilisé un cristal scintillateur $\mathrm{NaI}-\mathrm{Tl}$ et un photomultiplicateur EMI 5311, avec un appareillage électronique étudié au laboratoire de Chimie nucléaire du Collège de France par MM. Langevin, Allard et Corbé [8]; l'amplificateur proportionnel avait un gain maximum de 100 , le sélecteur différentiel permettait d'analyser un spectre d'impulsions entre 5 et ı $5 \mathrm{~V}$ avec une bande de largeur variable qui était en général de $2 \mathrm{~V}$.

Le premier photomultiplicateur utilisé dans cette étude était monté avec i I dynodes. Pour éviter la saturation avec les raies énergiques, nous devions appliquer entre étages une tension très inférieure à la normale, diminuant ainsi le pouvoir de résolution. Pour l'étude des coïncidences $\gamma \gamma$, nous avons monté un photomultiplicateur avec 9 étages seulement [9] et obtenu des résultats nettement meilleurs.

Il est nécessaire d'obtenir, par suite de la faible intensité des sources et de la longue période de ${ }^{126} \mathrm{I}$, une grande stabilité et un grand rendement du système de détection. Nous avons travaillé en géométrie non canalisée avec un gros cristal $(2,5 \times 2,5 \mathrm{~cm})$ fourni tout monté dans une boîte étanche par l'Harshaw-Chemical Co. Ce montage, adapté aux grandes photocathodes ne réalisait pas une bonne collection photonique pour l'EMI 5311. Nous avons en effet obtenu un meilleur pouvoir de résolution en montant pour l'étude des coïncidences $\gamma \gamma$, un cristal de mêmes dimensions avec diffuseur de magnésie, dans une boîte étanche ayant le diamètre de la photocathode.

Étude des rayonnements $\gamma$ ET X. - Etude des $\gamma$ énergiques. - On a obtenu avec une tension de $1250 \mathrm{~V}$ sur le photomultiplicateur le spectre d'impulsions

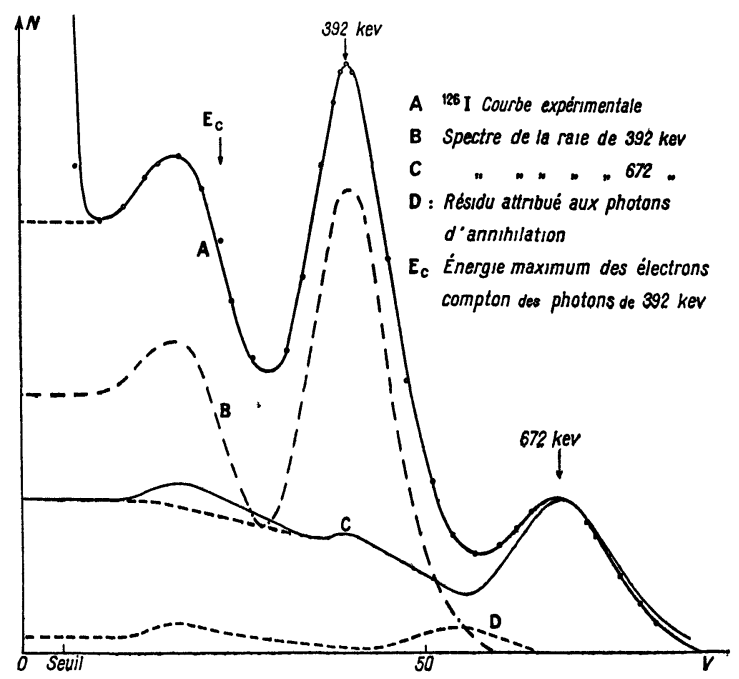

Fig. 4 .

représenté figure 4, pour la région des photons de 385 et $640 \mathrm{keV}$ précédemment signalés.

Pour obtenir une bonne précision et éliminer les défauts de linéarité du spectromètre, on a déterminé l'énergie des deux $\gamma$ mis en évidence par comparaison avec les raies de 4 i i $\mathrm{keV}$ de ${ }^{198} \mathrm{Au}$ et 66 I $\mathrm{keV}$ de ${ }^{137} \mathrm{Cs}$ [10] qui donnent des pics très voisins de ceux de ${ }^{126} \mathrm{I}$.

En bon accord avec les énergies déterminées au spectromètre à lentille, on a obtenu

$$
h v_{1}=672 \pm 7 \mathrm{keV}, \quad h v_{2}=392 \pm 5 \mathrm{keV} .
$$


Malgré une recherche systématique, aucun autre pic photoélectrique n'a été mis en évidence, ni dans la région supérieure à $672 \mathrm{keV}$, ni dans la région moyenne. Dans le domaine de 100 à $400 \mathrm{keV}$ une étude supplémentaire a été faite avec une tension de $1450 \mathrm{~V}$.

INTENSITÉ RELATIVE DES RAYONNEMENTS DE 672 ET $39^{2} \mathrm{keV}$. - On a comparé les surfaces des spectres simples attribuables dans la répartition d'impulsions de la figure 4 à l'absorption des photons de 392 et $672 \mathrm{keV}$ respectivement, compte tenu du rendement du cristal pour ces deux rayonnements.

$$
\frac{N_{\gamma 672}}{N_{\gamma 392}}=\frac{S_{\gamma 672}}{S_{\gamma 392}} \times \frac{\rho_{392}}{P_{672}} .
$$

Dans le domaine d'énergie considéré, la répartition d'impulsions à laquelle on doit s'attendre

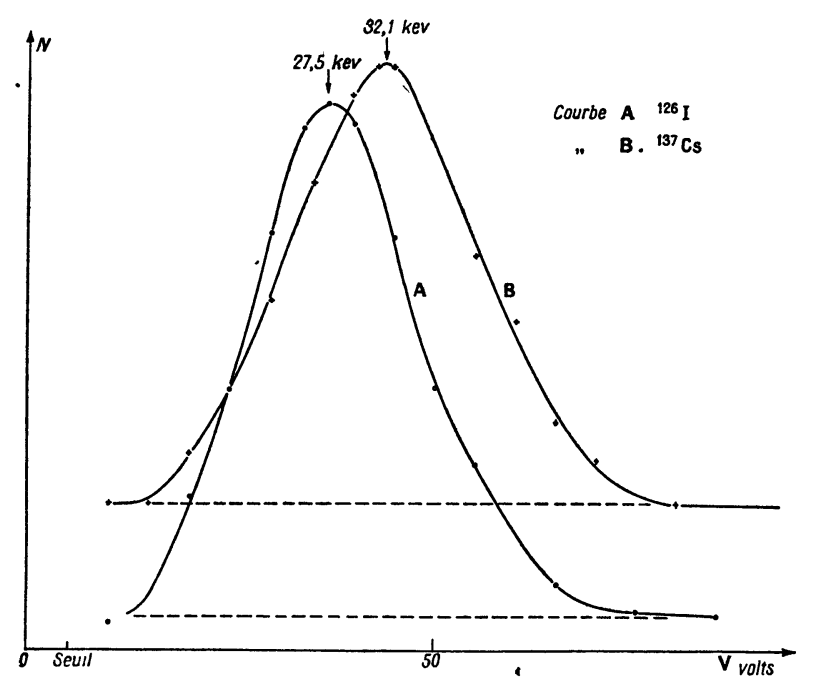

Fig. 5. - Rayonnements mous émis par ${ }^{126} \mathrm{I}$ et ${ }^{137} \mathrm{Cs}$.

pour des photons monoénergiques d'après l'absorption primaire photoélectrique et Compton est modifiée par des effets secondaires qui ne peuvent être négligés dans un gros cristal. Les photons Compton diffusés sont en partie réabsorbés dans le cristal, ce qui augmente l'importance de la raie photoélectrique, déforme et diminue le fond Compton. De plus le cristal absorbe des photons diffusés par la matière qui l'environne, en particulier par le verre situé devant la photocathode, provoquant une raie de " rétrodiffusion ". Maeder et Wintersteiger [11] ont calculé, dans le cas d'un cristal cylindrique de taille quelconque et d'un faisceau monoénergique canalisé, la forme du spectre en tenant compte de ces différents effets. Mais dans le cas d'un faisceau très ouvert, les calculs deviennent pratiquement inextricables.

On ne peut donc atteindre les spectres simples que par l'étude dans les mêmes conditions de $\gamma$ purs suffisamment voisins des $\gamma$ de ${ }^{126}$ I pour que l'on puisse admettre que la forme des spectres 'd'impulsions varient proportionnellement aux énergies.

On a obtenu les spectres des raies de $39^{2}$ et $67_{2} \mathrm{keV}$ par transformation des spectres de $4 \mathrm{I} \mathrm{I}$ et $66 \mathrm{I} \mathrm{keV}$ respectivement. Au préalable, on a supprimé la queue due au $\gamma$ de $680 \mathrm{keV}$ de ${ }^{198} \mathrm{Au}$ et dans les deux cas on a extrapolé le fond Compton dans la région déformée par les raies de fluorescence. [L'étude des basses énergies décrite plus loin indique que ce fond pour chacun des $\gamma$ reste pratiquement constant dans ce domaine (fig. 5)].

Quand on déduit du spectre de ${ }^{126} I$ (après extrapolation sous la raie $\mathrm{X}$ ) le spectre de $672 \mathrm{keV}$, on obtient un spectre différant peu de celui d'une raie pure de $392 \mathrm{keV}$. On rend compte presque rigoureusement du résidu par une faible proportion de photons d'annihilation. La décomposition du spectre total ainsi obtenue (fig. 4) confirme qu'il n'existe pas dans ce domaine de $\gamma$ supplémentaires plus intenses que le rayonnement de 5 1 o keV. Ayant retranché la raie de "rétrodiffusion", on a obtenu le rapport des nombres de photons absorbés dans le cristal

$$
\frac{n_{1}}{n_{2}}=\frac{S_{672}}{S_{392}}=0,68 .
$$

On a calculé dans la géométrie utilisée le rendement du cristal pour chacun des $\gamma$ par intégration graphique. Les coefficients d'absorption totale dans INa $\left(\mu_{392}=0,435 \mathrm{~cm}^{-1}\right.$ et $\left.\mu_{612}=0,275 \mathrm{~cm}^{-1}\right)$ ont été calculés d'après les valeurs de Davisson et Evans [12]. Les rendements obtenus sont

$$
\rho_{2}=0,102, \quad \rho_{1}=0,067, \quad \frac{\rho_{2}}{\rho_{1}}=1,52 ;
$$

on n'a tenu compte ni du diamètre de la source $(5 \mathrm{~mm})$ ni de l'absorption des photons dans l'écran d'aluminium, l'erreur commise étant négligeable devant celle qui affecte le rapport $\frac{S_{1}}{S_{2}}$. Nous obtenons alors

$$
\frac{N_{\gamma 672}}{N_{\gamma 392}}=\frac{S_{672}}{S_{392}} \times \frac{\rho_{392}}{\rho_{672}}=1,02 \pm 0,1 .
$$

Étude DE LA RÉGION DE FAIBLE ÉNERGIE. Le spectre de basse énergie de ${ }^{126} I$ représenté figure 5 a été obtenu avec une tension de $1800 \mathrm{~V}$ et en remplaçant l'écran d'aluminium par $3,5 \mathrm{~mm}$ de plexiglass pour diminuer l'absorption des photons mous. On a déterminé l'énergie de la raie molle qui se détache sur le fond des $\gamma$ énergiques, par comparaison avec la raie $\mathrm{X}$ obienue avec ${ }^{137} \mathrm{Cs}$ dans les mêmes conditions. En prenant pour le sommet de la raie l'énergie des raies $K_{\alpha}$ du baryum soit, $32, \mathrm{r} \mathrm{keV}$, on trouve pour le pic de ${ }^{126} \mathrm{I}$ une énergie de $27 \pm 0,5 \mathrm{keV}$ compatible avec l'énergie des $K_{\alpha} \mathrm{du}$ tellure $(27,5 \mathrm{keV})$ et non du xénon $(29,7 \mathrm{keV})$. La raie molle provient donc de la capture $K$ 
signalée [2] et non d'une conversion interne dans la branche $\beta-$.

RAPPORT D'INTENSITÉ $\frac{\text { Capture } K}{\gamma_{672}}$ - Les énergies des deux rayonnements étant très différentes, nous avons employé une méthode indirecte utilisant la connaissance du rapport $\frac{N_{\mathrm{X}}}{N_{\gamma}}$ dans le cas de ${ }^{137} \mathrm{Cs}$. De la comparaison de l'intensité des $\mathrm{X}$ d'une part, des $\gamma$ énergiques d'autre part, de ${ }^{126}$ I et ${ }^{137} \mathrm{Cs}$ on peut alors déduire le rapport $\frac{\text { Capture } K}{N_{\curlyvee}}$.

$$
\frac{\text { Capture } K}{N_{\gamma 672}}=\frac{\omega_{K} \mathrm{Ba}}{\omega_{K} \mathrm{Te}} \alpha_{K}\left(\frac{N_{\mathrm{X}} \mathrm{I}}{N_{\mathrm{X}} \mathrm{Cs}}\right) \times\left(\frac{N_{Y} \mathrm{Cs}}{N_{\gamma 672} \mathrm{I}}\right) .
$$

Les valeurs des facteurs de fluorescence sont d'après la formule empirique de Burhop [13] :

$$
\omega_{K} \mathrm{Ba}=0,895, \quad \omega_{K} \mathrm{Te}=0,865 ;
$$

d'autre part,

$$
\alpha_{K}=0,097 \quad[14]
$$

Le rapport $\frac{N_{\gamma} \mathrm{Cs}}{N_{\gamma_{672} I}}$ a été pris égal au rapport des hauteurs des pics photoélectriques.

Avec des sources intenses, par suite de la présence d'un grand nombre de $\gamma$ énergiques produisant des queues d'impulsions négatives importantes, nous avons obtenu des spectres déformés pour les raies $\mathrm{X}$. Avec des sources d'intensité faible et analogue, nous avons obtenu les répartitions de la figure 5. Le rapport d'intensité des $\mathrm{X}$ est pris égal au rapport des surfaces des raies en tenant compte de l'absorption des photons et de l'efficacité pratique du cristal (tableau I).

\section{TABLEAU I.}

$t=\frac{\text { Nombre de photons atteignant le cristal }}{\text { Nombre de photons émis dans l'angle } 4 \pi}$.

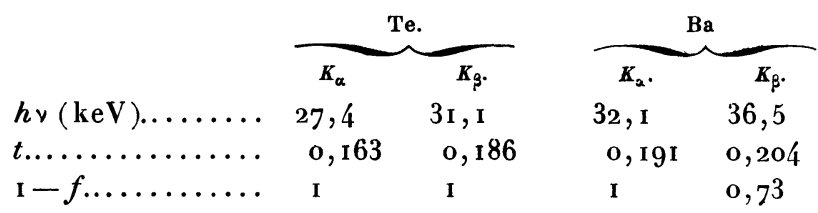

L'absorption de ces rayonnements mous dans $\mathrm{NaI}$ est ioo pour 1oo. Cependant les raies $K_{\beta}$ du baryum, qui ont une énergie supérieure au saut d'absorption $K$ de l'iode donnent, à côté de la raie d'énergie totale, une raie fantôme $\left(h \nu K_{\beta}\right)_{B a}-(h \nu K)_{1}$ d'énergie trop faible pour être détectée. Si $f$ est la fraction des photons incidents donnant ce pic, l'efficacité pratique est $(\mathrm{I}-f)$.

Pour un faisceau normal, on obtient facilement

$$
f=\frac{1}{2} \omega_{K_{1}}\left(1-\frac{\mu_{K}}{\tau} L \frac{\tau+\mu_{K}}{\mu_{K}}\right),
$$

où $\mu_{\Lambda}$ et $\tau$ sont les coefficients d'absorption des raies $K$ de l'iode et du rayonnement incident respectivement dans INa ( $\left.{ }^{2}\right)$.

Si le faisceat fait un angle $\theta_{0}$ avec la normale, il suffit de remplacer $\tau \operatorname{par} \frac{\tau}{\cos \theta_{0}}$. On obtient ainsi des limites inférieures et supérieures du facteur $f$ pour un faisceau non canalisé pour $\left(h \nu K_{\beta}\right)_{\mathrm{Ba}}$. D'où

$$
f=0,27 \pm 0,05 \text {. }
$$

Sachant que le rayonnement $X$ se compose de 75 pour 1 oo de raies $K_{\alpha}$ et 25 pour 1 oo de raies $K_{\beta}$ [15], on ontıent en utilisant les valeurs du tableau I :

$$
\frac{N_{\mathrm{X}} \mathrm{I}}{N_{\mathrm{X}} \mathrm{Cs}}=\frac{0,180}{0, \mathrm{I} 69} \frac{S_{\mathrm{X}} \mathrm{I}}{S_{\mathrm{X}} \mathrm{Cs}} \quad \text { et } \quad \frac{N_{\mathrm{capt} K}}{N_{\gamma_{672}}}=\mathrm{I}, 35 \pm 0, \mathrm{I} .
$$

Mesures en coincidence. - Nous avons étudié les coïncidences $\beta \gamma, X \gamma$ et $\gamma \gamma$. Dans les deux premiers cas, les impulsions produites par un premier détecteur servaient à " déverrouiller " l'amplificateur du spectromètre à scintillation pendant $210^{-6} \mathrm{~s}$ [8]. Dans le troisième, nous avons étudié les coïncidences entre les impulsions provenant de deux spectromètres à scintillation. Le temps de résolution du système à coïncidence était environ de $5 \mathrm{IO}^{-7} \mathrm{~s}$.

ÉTUde des coïncidences $\beta \gamma$. - Les $\beta$ étaient détectés par un compteur cloche à fenêtre de mica

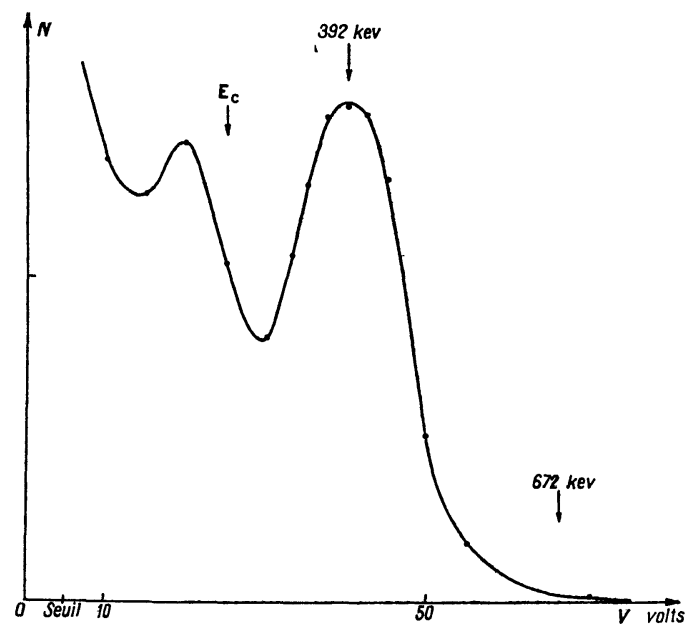

Fig. 6. - Spectre $\gamma$ de ${ }^{128} I$ en coïncidence avec le rayonnement $\beta$.

de $3 \mathrm{mg} / \mathrm{cm}^{2}$ placé à quelques centimètres de la source.

On a d'abord étudié le spectre des photons en

(2) Cette formule ne diffère de celle donnée par $T$. B. Novey (Phys. Rev., 1953, 89, 672) que par l'introduction du facteur de fluorescence. 
coïncidence avec les $\beta$. Dans la région molle, aucune raie n'a été mise en évidence. Le spectre d'impulsions obtenu pour les $\gamma$ énergiques (fig. 6) permet d'affirmer : $a$. que le rayonnement॰de $39^{2} \mathrm{keV}$ est en coïncidence avec les $\beta^{-} ; b$. que le rayonnement de $672 \mathrm{keV}$ n'appartient pas à la branche $\beta^{-}$; c. qu'aucun $\gamma$ d'intensité notable, d'énergie supérieure à $500 \mathrm{keV}$ n'existe dans la branche $\beta^{-}$.

L'étude de l'absorption des $\beta$ en coïncidence avec la raie de $392 \mathrm{keV}$ par comparaison avec l'étude d'une source de ${ }^{198} \mathrm{~A} u$ montre que : $a$. le rapport $\frac{N_{\beta \gamma}}{N_{\beta}}$ n'étant pas constant, la raie de $39^{2} \mathrm{keV}$ n'est pas en cascade avec l'ensemble des $\beta$; $b$. la valeur de l'absorption moitié du spectre en coïncidence comparée à celle du spectre de $970 \mathrm{keV}$ de $\mathrm{Au}$ est compatible avec l'énergie maximum de $870 \mathrm{keV}$ du deuxième spectre $\beta$ - déterminée au spectromètre à lentille.

CoïnCidences $X-\gamma_{672}$. - Nous avons utilisé pour la détection des $\gamma$ énergiques, un deuxième cristal INa-Tl avec un photomultiplicateur EMI 5311 suivi d'un simple discriminateur d'amplitude permettant de compter exclusivement les impulsions de la raie de $672 \mathrm{keV}$.

Les impulsions de ce système servaient à " déverrouiller " le spectromètre réglé pour l'étude de la région de faible énergie. Le spectre en coincidence met en évidence la même raie $X$ que le spectre direct, avec un rapport $\frac{\text { hauteur de la raie }}{\text { fond }}$ nettement supérieur.

Les coincidences fortuites donneraient une répartition différente, l'amplificateur se débloquant dans ce cas au hasard par rapport au début de l'impulsion $\mathrm{X}$. Le rayonnement de $672 \mathrm{keV}$ appartient donc bien à la branche capture $K$.

CoÏncidences $\gamma \gamma$. Des coïncidences $\gamma \gamma$ ayant été signalées précédemment [2], nous avons repris cette étude avec deux sélecteurs différentiels et un circuit à coỉncidence de temps de résolution environ $5 \cdot 10^{-7} \mathrm{~s}$. La source était placée à faible distance de chacun des cristaux, des écrans étant interposés pour arrêter les rayons $\beta$. La source d'iode dont nous disposions à ce moment étant très faible, les coincidences fortuites étaient négligeables.

Nous avons tout d'abord repris l'étude des coinncidences $X-\gamma$, un des sélecteurs étant réglé sur la raie $X$, l'autre balayant la région énergique. La répartition des coïncidences confirme l'attribution du $\gamma$ de $672 \mathrm{keV}$ à la branche capture $K$, de même que la non-existence de $\gamma$ notables supplémentaires dans cette branche.

Nous avons ensuite cherché à mettre en évidence des coincidences entre la raie de $39^{2} \mathrm{keV}$ et un éventuel $\gamma$ de $400 \mathrm{keV}$ (ce cas se présentant dans l'hypothèse d'un troisième spectre $\beta^{-}$). Le spectre de colncidence obtenu en se plaçant sur le pic de $39^{2} \mathrm{keV}$ pour le premier selecteur présente en fait un maximum dans la région de $500 \mathrm{keV}$.

En plaçant le sélecteur sur $500 \mathrm{keV}$, nous n'avons pas retrouvé la même répartition, mais un fond sur lequel se détache un pic vers $180 \mathrm{keV}$. Un photon de $672 \mathrm{keV}$ ayant projeté dans le premier cristal un électron Compton d'énergie maximum (49o keV) est diffusé en arrière avec une énergie de $182 \mathrm{keV}$; il a une grande probabilité d'être absorbé dans le deuxième cristal très proche, donc de produire une impulsion correspondant à $180 \mathrm{keV}$ en coïncidence avec l'impulsion Compton transmise au premier sélecteur.

Les spectres de coïncidences peuvent s'interpréter dans les deux cas comme un mélange de coïncidences dues aux photons d'annihilation et aux $\gamma$ diffusés.

Les taux de coïncidences obtenus, comparés aux coïncidences $X-\gamma$ excluent de toute façon une cascade $390-390 \mathrm{keV}$ de plus de ıo pour ıoo, aucune coïncidence vraie n'a été trouvée avec le $\gamma$ de $672 \mathrm{keV}$ dans la région des $\gamma$ énergiques.

3. Établissement et discussion du schéma de désintégration. - Les résultats obtenus à l'aide

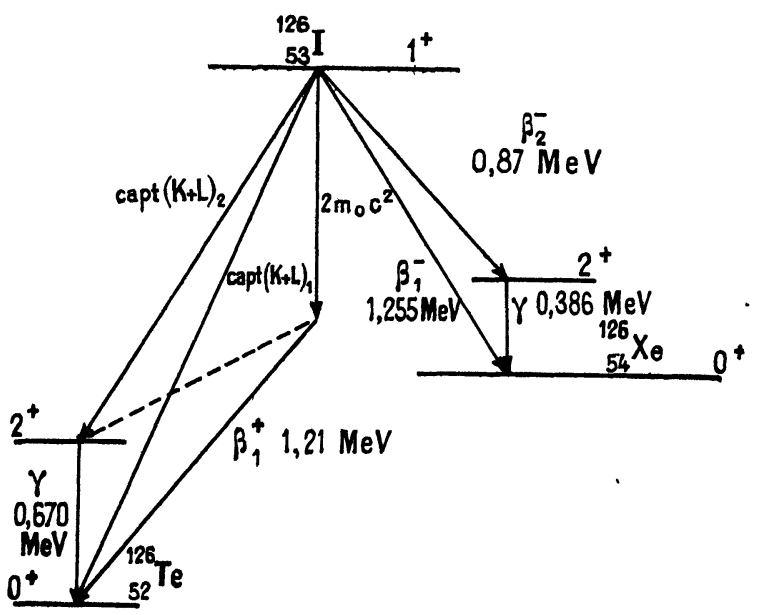

Fig. 7. - Schéma de désintégration de ${ }^{126} \mathrm{I}$.

du spectromètre à lentille magnétique et des compteurs à scintillation nous permettent d'établir un schéma cohérent pour la désintégration de ${ }^{126} \mathrm{I}$ (fig. 7). La différence entre les énergies maxıma des deux spectres $\beta$ - et l'obtention de coincidences $\beta$ photon de $386 \mathrm{keV}$, conduisent à un schéma de niveaux pour ${ }^{126} \mathrm{Xe}$ en accord avec les résultats précédents [1], [2].

Nous avons montré pour la désintégration par capture $K$ qu'une grande partie :des rayons $\mathrm{X}$ était en coïncidence avec le photon de $670 \mathrm{keV}$; nous attribuons le reste des rayons $\mathrm{X}$ à une deuxième capture $K$ aboutissant au niveau fondamental 
de ${ }^{126}$ Te. La faible intensité des spectres d'électrons positifs ne nous a pas permis d'affirmer l'existence ou la non-existence de coincidence $\beta^{+} \gamma$, par contre étant donnée l'énergie maxima élevée du spectre $\beta^{+}$ et la valeur des rapports $\frac{\text { Capture } K}{\beta^{+}}$obtenus en supposant que le spectre d'électrons positifs aboutit soit au niveau excité, soit au niveau fondamental de ${ }^{126} \mathrm{Te}$, nous avons conclu à la solution représentée sur le schéma.

Le tableau II donne les valeurs des intensités partielles $I$ et des probabilités de désintégration $f$ pour les différentes transitions.

Tableau II.

\begin{tabular}{|c|c|c|c|c|c|}
\hline & $I$ & $f$ & $t$ & $f t$ & $\log f t$ \\
\hline & $o, 129$ & 20 & $8,7 \cdot 10^{6}$ & $\mathrm{I}, 74 \cdot 10^{8}$ & 8,24 \\
\hline$\cdots$ & o, 338 & 79,6 & $3,33 \cdot 10^{6}$ & $2,65 \cdot 10^{8}$ & 8,42 \\
\hline apture $K_{1} \ldots$ & $o, 157$ & I 3,4 & $7,15 \cdot 10^{6}$ & $9,55 \cdot 10^{7}$ & $7,9^{8}$ \\
\hline apture $K_{2} \ldots$ & 0,305 & 6,24 & $3,69 \cdot 10^{6}$ & $2,3 \cdot 10^{7}$ & 7,36 \\
\hline a & 0,0125 & 3,17 & $.10^{7}$ & $2,86 \cdot 10^{8}$ & 8,45 \\
\hline
\end{tabular}

Les intensités partielles données dans le schéma sont obtenues en estimant que toute capture $K$ est accompagnée d'une capture $L$ telle que

$$
\frac{\text { Capture }(K+L)}{\text { Capture } K}=\text { I , I2, }
$$

d'après les valeurs théoriques de Rose et Jackson [16]. L'intensité du rayonnement $\gamma$ de $670 \mathrm{keV}$ donne la somme des intensités des désintégraxions par capture $K$ et $L$. On déduit le rapport

$$
\frac{\text { Capture }(K+L) \text { total }}{\beta-\text { total }}=\mathrm{I}, \mathrm{I} \pm 0, \mathrm{I} 5,
$$

valeur inférieure à la valeur de r,44 donnée par Perlman et Friedlander [2]; cet écart peut s'expliquer par le fait que dans notre cas, seul intervient le rapport des facteurs de fluorescence $\omega$ pour les rayonnements $\mathrm{X}$ de $\mathrm{Te}$ et de $\mathrm{Ba}$, alors que pour les mesures de Perlman et Friedlander, il faut tenir compte de la valeur absolue de $\omega_{\mathrm{Te}}$; elle est choisie par ces auteurs comme égale à 0,75 ; si l'on prend la valeur de o,85 donnée par Burhop [13] pour $Z=52$, on trouverait un rapport $\frac{\text { Capture } K}{\beta-}$ se rapprochant de celui que nous trouvons.

Les valeurs $f\left(f^{+}\right.$et $\left.f^{-}\right)$des probabilités de désintégration par émission $\beta^{+}$et $\beta^{-}$sont tirées des courbes données par Feenberg et Trigg [17] dans le cas des transitions permises; pour la capture $K$, nous avons calculé directement $f_{k}$ en u uilisant la formule correspondant aux transitions permises

$$
f_{K}=2 \pi(\alpha Z)^{2 s+1}(2 R)^{2 s-2} \frac{\mathrm{I}+s}{\Gamma(2 s+\mathrm{I})}\left(W_{0}+s\right)^{2},
$$

où $R$ et $Z$ sont le rayon et le numéro atomique du noyau qui se désintègre, $\alpha$ la constante de structure fine, $s=\sqrt{1-\alpha^{2} Z^{2}} ; W_{0}$ représente en unités $\mathrm{mc}^{2}$ l'énergie totale maxima d'un electron lors d'une émission $\beta^{+}$qui aboutirait au même niveau que la capture $K$.

RAPport $\frac{\text { Capture } K}{\beta^{+}}$. Dans le tableau III nous comparons les valeurs expérimentales et théoriques des probabilités de désintégrations par capture $K$ et émission $\beta^{+}$aux niveaux fondamental et excité de ${ }^{126}$ Te. Nos valeurs expérimentales sont obtenues en considérant que les rayons $\mathrm{X}$ proviennent

\begin{tabular}{|c|c|c|c|c|}
\hline Niveaux de ${ }^{126} \Gamma e$. & $\left(\frac{\text { Capture } K}{\beta+}\right)_{\text {esp }}$. & $\begin{array}{l}\text { Transitions } \\
\text { permıses }\end{array}$ & $\begin{array}{l}\Delta J=1, \\
\Delta l=2 .\end{array}$ & $\begin{array}{c}\Delta J=2 \\
\text { our }\end{array}$ \\
\hline Fondamental. & $\mathrm{I} 2,5\left\{\begin{array}{l}+7 \\
-3\end{array}\right\}$ & 4,25 & 3,5 & I 8 \\
\hline $670 \mathrm{keV}$. & $>75$ & 62 & 55 & - \\
\hline
\end{tabular}
tous des captures $K_{1}$ et. $K_{2}$.

Tableau III.

Nous avons porté dans le tableau les valeurs théoriques du rapport $\frac{\text { Capture } K}{\beta^{+}}$pour les transitions interdites $(\Delta J=\mathrm{I}, \Delta l=2)$ et $\Delta J=2$, oui qui comme nous le verrons par la suite peuvent expliquer les résultats trouvés pour le spectre $\beta^{+}$émis par ${ }^{126} \mathrm{I}$. Les rapports de probabilités de désintégration par émission $\beta^{+}$et capture $K$ pour une transition interdite $(\Delta J=1, \Delta l=2)$ et une transition permise sont, d'après R. Nataf et R. Bouchez [18],

$$
\begin{gathered}
\frac{f_{K}(\Delta J=\mathrm{I}, \Delta l=2)}{f_{K} \text { permis }}=\left[\sqrt{\frac{\mathrm{I}-s}{\mathrm{I}+s}}-\left(W_{0}+s\right) \frac{R}{3}\right]^{2}, \\
\frac{f^{+}(\Delta J=\mathrm{I}, \Delta l=2)}{f^{+} \text {permıs }}=\frac{\mathrm{I}-\gamma_{0}}{2} \quad \text { où } \quad \gamma_{0}=\sqrt{\mathrm{I}-\alpha^{2} Z_{\mathrm{eff}}^{2}}
\end{gathered}
$$

$Z_{\text {eff }}$ étant le numéro atomique du noyau formé par l'émission $\beta^{+}$.

Les valeurs expérimentales sont supérieures aux valeurs théoriques dans le cas $(\Delta J=1, \Delta l=2)$; les limites inférieure pour le niveau excité et supérieure pour le niveau fondamental données pour $\frac{\text { Capture } K}{\beta^{+}}$proviennent du fait que lors de la décomposition du spectre $\beta^{+}$par la méthode des droites de Fermi l'existence du deuxième spectre $\beta^{+} d^{\prime}$ 'énergie maxima $0,5 \mathrm{MeV}$ ne peut être exclue, mais on peut affirmer que son intensité est inférieure à la moitié du spectre $\beta^{+}$aboutissant au niveau fondamental. Jusqu'à présent, si l'accord entre les valeurs expérimentales et théoriques semble bon dans le cas de transitions permises [19], dans le cas de transitions interdites les résultats peu nombreux et peu précis ne permettent pas de conclure. 
NATURE DES RAYONNEMENTS $\gamma$ - - Le coefficient de conversion obtenu pour le photon de $386 \mathrm{keV}$ était $\alpha_{2}=(1,65 \pm 0,2) \cdot 10^{-2}$; cette valeur est en bon accord avec celle donnée dans les tables de Rose et al. [20] pour un quadrupole électrique $\alpha_{2}=1,63.10^{-2}$, la valeur donnée pour un dipole magnétique étant $\beta_{1}=2,1.10^{-2}$. Le rapport $\frac{\alpha_{K}}{\alpha_{L}}$ des coefficients de conversion dans les couches $K$ et $L$ a été trouvé supérieur à 8 (fig. 3 ). Une courbe empirique de la valeur de ce rapport en fonction de $\frac{Z^{2}}{E}$, où $E$ est l'énergie du photon en kiloélectror-volts, a été donnée par Goldhaber et Sunyar [21] jusqu'à une valeur de $\frac{Z^{2}}{E} \sim 9,3$ qui donne $\frac{\alpha_{K}}{\alpha_{L}}=5,12$; la limite inférieure obtenue pour le photon de $386 \mathrm{keV}$ de ${ }^{126} \mathrm{Xe}$ est compatible avec une extrapolation de cette courbe jusqu'à une valeur $\frac{Z^{2}}{E}=7,55$.

La nature de quadrupole électrique du photon de $386 \mathrm{keV}$ confirme l'attribution du spin $2_{2}$ et de la parité pair au premier etat excité de ${ }^{126} \mathrm{Xe}$.

On peut supposer que le photon de $670 \mathrm{keV}$ est lui aussi un quadrupole électrique, son coefficient de conversion serait alors $3.10^{-3}$ avec une raie d'électrons de conversion de largeur à mi-hauteur environ double de celle de la raie de $386 \mathrm{keV}$, donc à la limite de ce que nous pouvons séparer des spectres continus $\beta$.

DÉTERMINATION DU SPIN ET DE LA PARITÉ DES NIVEAUX DE ${ }^{126} \mathrm{I}$, ${ }^{126} \mathrm{Te}$ ET ${ }^{126} \mathrm{Xe}$. - D'après les valeurs de $f t$, les diverses transitions $\beta$ sont soit interdites d'ordre $\mathbf{I}$ avec changement de parité, soit interdites en $l(\Delta J=1, \Delta l=2)$ sans changement de parité [22], [231; ${ }^{126} \mathrm{Te}$ et ${ }^{126} \mathrm{Xe}$ étant des noyaux pair-pair, leur niveau fondamental est pair avec un spin nul; leur premier état excité est vraisemblablement pair avec un spin égal à 2 .

${ }^{126} 1$ se compose de 53 protons et de 73 neutrons; d'après le modèle en couche de Mayer-Jensen [24] et les spins connus des noyaux stables [25] les états les plus probables pour le proton et le neutron non couplés sont les suivants :

$$
\begin{array}{ccccc}
p \ldots \ldots \ldots & g_{7 / 2} & d_{5 / 2} & - & - \\
n \ldots \ldots & s_{1 / 2} & d_{3 / 2} & g_{7 / 2} & h_{11 / 2}
\end{array}
$$

L'état $h_{11 / 2}$ est d'après M. Mayer peu probable en rasson de la tendance des nucléons à spin élevé à se coupler deux à deux; seul cet état $h_{\mathrm{i} 1 / 2}$ du neutron entraîne pour l'état fondamental de ${ }^{126} \mathrm{I}$ une parité - quel que soit l'etat du proton, les autres états possibles pour le neutron donnant une parité + .

Nordheim [23] attribue à ${ }^{126}$ I l'état $\left(d_{5 / 2}, g_{7 / 2}\right)$; Stevenson et Deutsch [26] par comparaison des mesures de corrélation angulaire faites avec ${ }^{126} \mathrm{I}$ et ${ }^{86} \mathrm{Rb}$ et attribuant au spectre énergique $\beta^{-}$émis par 126I une forme interdite donnent un état $2^{-}$. Pour un état $2^{-}\left(g_{7 / 2}, h_{11 / 2}\right)$ de ${ }^{126}$ I les transitions $\beta_{1}^{-}, \beta_{1}^{+}$et capture $K_{1}$ seraient une fois interdites avec $\Delta J=2$, les spectres différant de la forme permise por le facteur

$$
\alpha=p^{2}+q^{2}=\left(W^{2}-\mathrm{I}\right)+\left(W_{0}-W\right)^{2} ;
$$

les transitions $\beta_{2}^{-}$et (capt. $\left.K\right)_{2}$ serarent des transitions $\Delta J=0$ oui, donc aussi interdites d'ordre I aves une forme permise pour $\beta_{2}^{-}$.

Dans le tableau IIl, le rapport théorique $\frac{\text { Capture } K}{\beta^{+}}$ pour une transition $\Delta J=2$ oui est plus voisin de la valeur trouvée expérimentalement que les rapports $\frac{\text { Capture } K}{\beta^{+}}$pour des transitions permises ou interdites en $\mathbf{I}$.

Nous avons, d'autre part, essayé de décomposer les spectres $\beta$ - par la méthode de la droite de Fermi en appliquant le facteur de correction $\alpha$ au spectre le plus énergique : il est impossible d'obtenir une droite en choisissant comme énergie maxima du spectre $\beta_{1}^{-}$une valeur inférieure à celle donnée par extrapolation de la droite de Fermi tracée pour un spectre de forme permise; après soustraction du spectre le plus énergique, on ne retrouve pas pour les spectres $\beta_{2}^{-}$une forme permise. La différence des énergies maxima des deux spectres est alors de l'ordre de $325 \mathrm{keV}$, donc très inférieure à celle trouvée pour la décomposition des spectres (fig. 2) qui était en bon accord avec l'énergie de $386 \mathrm{keV}$ du photon émis en cascade avec $\beta_{2}^{-}$. De plus, la valeur de $\log f t(8,42)$ trouvée pour le spectre $\beta_{2}^{-}$ est trop élevée par rapport aux valeurs habituelles pour les transitions $\Delta J=0,1$ oui [23]. Ceci nous conduit à choisir pour l'état fondamental de ${ }^{126}$ I un état $\mathrm{I}^{+}$de préférence à un état $2^{-}$; l'état $\mathrm{I}^{+}$peut être du à l'une des trois configurations $\left(g_{7 / 2} g_{7 / 2}\right)$, $\left(d_{\mathrm{s} / 2} d_{3 / 2}\right),\left(d_{5 / 9}, g_{7 / 2}\right)$, les deux dernières étant plus vraisemblables, car en accord avec la règle de Nordheim [23].

D'après la théorie [19], les spectres $\beta$ interdits en $l$ ont des formes voisines de la forme permise, ne s'écartant de cette forme que de quelques pour-cent et pour des énergies assez basses.

L'épaisseur des sources que nous avons utilisées dans l'étude des spectres $\beta$ apportait une distorsion supérieure à quelques pour-cent et se faisant sentir pour des énergies inférieures à $500 \mathrm{keV}$, ce qui suffit à masquer tout écart du spectre par rapport à la forme permise.

Tous les résultats obtenus peuvent donc s'expliquer par le schéma (fig. 7), le seul écart important par rapport aux données théoriques étant le rapport $\frac{\text { Capture } K}{\beta^{+}}$pour la transition à l'état fondamental de ${ }^{126} \mathrm{Te}$.

Ce travail a été effectué grâce à l'aide financière 
du Centre National de la Recherche Scientifique et sous la direction de M. le Professeur F. Joliot que nous tenons à remercier pour l'intérêt qu'il luı a apporté. Nous remercions également l'équipe du synchrocyclotron Philips d'Amsterdam et tout particulièrement $M$. Van Atten Jr. pour les sources de ${ }^{126}$ I qu'il a bien voulu nous préparer. Enfin nous sommes reconnaissants à MM. Langevin, Allard et Corbé qui ont réalisé et mis au point les montages électroniques utilises au cours de cette étude.

$$
\text { Manuscrit reçu le } 2 \text { juin } 1953 .
$$

\section{BIBLIOGRAPHIE.}

[1] Mitchell A. C. G., Mei J. Y., Maienschein F. C. et Peacock C. L. - Phys. Rev., 1949, 76, 1450.

[2] Perlman M. L. et Friedlander G. - Phys. Rev., $1951,82,440$.

[3] Nordheim L. W. - Rev. Mod. Physics, r 95 ז, 23, 322.

[4] Marty N., Langevin H. et Hubert P. - C. R. Acad. Sc., 1 953,236, I 53.

[5] Hubert P. - J. Physique Rad., i 951, 12, 763.

[6] Hubert P. - Thèse à paraitre aux Annales de Physıque.

[7] Hornyak W., Lauritsen T. et Rasmussen V. K. Phys. Rev., , 1949, 76, 73 г.

[8] Langevin M., Allard G. et Corbé G. - J. Physique Rad., I 953, 14, 69 I.

[9] Robins R. - Proc. Phys. Soc., i 952 , B 65, 320.

[10] Muller D. E., Hoyt H. C., Kleim D. J. et Du Mond S. W. M. - Phys. Rev., I 952, 88, 775 .

[11] Maeder D., Wintersteiger V. - Phys. Rev., ig52, 87, 537; Helv. Phys. Acta, 1952, 25, 465; Communication privée.

[12] Davisson C. M. et Evans R. O. - Rev. Mod. Physics, 1 $952,24,79$.

[13] Burhop E. M. S.-The Auger Effeet. Cambridge University Press, i 952, p. 48.
[14] Health R. L. et Bell P. R. - Phys. Rev., i 952 , 87, I 76 ; Waggoner N. A. - Phys. Rev., 1951, 82, 906.

[15] Manne Siegbahn. - Upsala, i 952 , p. 354-355.

[16] Rose M. E. et Jackson J. L. - Phys. Rev., 1949, 76, 1540.

[17] Feenberg E. et Trigg G. - Rev. Mod. Physics, i95o, 22, 399 .

[18] Nataf R. et Bouchez R. - J. Physique Rad., i952, 13, 190.

[19] Bouchez R. - Physica, 1952, 18, I I 7 I.

[20] Rose M. E., Goertzel G. H., Spinrad B. I., Harr J. et Strong P. - Phys. Rev., i $95 \mathrm{r}, 83,79$.

[21] Goldhaber M. et Sunyar A. W. - Phys. Rev., r95i, 83, 906.

[22] Mayer M. G., Moszkowski S. A. et Nordheim L. W. Rev. Mod. Physics, i $951,23,3$ ז 5 .

[23] Nordheim L. W. - Rev. Mod. Physics, r 95 ז, 23, 322.

[24] Mayer M. G. - Phys. Rev., 1949, 75, 1969; 1950, 78, 16.

[25] Klinkenberg P. F. A. - Rev. Mod. Physics, 1952, 24, 63.

[26] Stevenson D. T. et Deutsch M. - Phys. Rev., г 95 r, 84, 1071 . 